\title{
Interactive comment on "Deciphering the evolution of the Bleis Marscha rock glacier (Val d'Err, eastern Switzerland) with cosmogenic nuclide exposure dating, aerial image correlation, and finite-element modelling" by Dominik Amschwand et al.
}

\section{Dominik Amschwand et al.}

dominik.amschwand@unifr.ch

Received and published: 27 November 2020

Dear Jakob Heyman, Dear Leif Anderson, Dear Andreas Vieli, editor of The Cryosphere

We sincerely thank you and reviewers Jakob Heyman and Leif Anderson for considering our paper for publications in The Cryosphere. We thank both reviewers for their positive comments on our approach combining diverse methodologies to understand 
the evolution of the Bleis Marscha rock glacier. This paper is one of the first to use cosmogenic nuclides on a fully active rock glacier. We obtained early Holocene exposure ages from boulders close to the toe of the rock glacier and ages of only a few hundred years close to the root zone. Both reviewers brought up questions on our interpretation of the exposure ages, notably that we used a different approach in interpreting the ages on the upper, more active lobe than on the lower lobe. These comments have led us to re-evaluate our interpretation of the ages on the lower lobe. In that light, we are convinced that the input from the two reviewers will lead to an improved paper and we look forward to having the opportunity to revise our manuscript along the lines suggested by the reviewers. Below we summarize the concerns brought up by the two reviewers and outline our planned approach to revising the paper. A number of points were raised by both reviewers. Comments relating to each specific review are given further below.

Both reviewers note that we have used different approaches to interpreting exposure ages for the upper versus the lower lobe. We thank the reviewers for pointing this out and will use a consistent approach in the revised version. Further details on this are given in our comments below on Reviewer 1's suggestions. In a similar vein, both reviewers point out that our thoughts on how exposure ages on active rock glaciers should be interpreted should be included. In a revised version, we will include a few sentences on what processes lead to too old ages and what lead to too young ages on a moving rock glacier. Adding such content to our manuscript will be an enlightened and incisive addition given that our ages actually cannot be interpreted as only time elapsed since stabilization, as in previous publications. Such a discussion will proceed specifically in light of the Bleis Marscha site.

Comments by Reviewer 2 additionally prompted a reassessment of the level of detection of the surface velocities from image correlation and clarifications on the assumptions embedded in the numerical model. We thank Reviewer 2 for these suggestions and will include these in the revised manuscript. See detailed response below.

Printer-friendly version

Discussion paper

Finally, Reviewer 2 states "It would be great if the surface velocity estimates, the field 
observations, and the model could be better used to justify the interpretation of the surface exposure ages. Right now, the manuscript seems to touch on these different features and then transitions into the Holocene history of the Bleis Marscha rock glacier without too clear of a connection between the modern and paleo perspectives. It would better honor all the work in the manuscript if logic behind the assumptions being made was laid out in connection to the modern analysis of the rock glacier. This is no easy task, but I think one that will really highlight the broad scope of methods brought to bear in this body of work." In a revised version we will pay close attention to weaving the various different threads, geomorphology, isotopic dates and numerical modelling, together. Additionally, Reviewer 2 noted that the presence of unnecessary details leads to a digression from the main message, that the connection between observations/results and the interpreted Bleis Marscha rock-glacier history is not clear enough and that important thoughts on the interpretation of the exposure ages are not properly spelled out. Therefore, we will condense parts of the manuscript and move some of the details into a supplemental section. The re-interpretation of the ages on the lower lobe triggered by the reviewers' criticisms enables substantial simplifications in the revised manuscript. By spelling out the age model we also hope to clarify the connection between observations on Bleis Marscha and the interpreted rock-glacier evolution scenario. Both reviewers made a number of suggestions for improving the figures. These and all minor line-by-line points made by the reviewers will be taken into account in a revised version. Finally, as suggested by Reviewer 2, a roadmap that relates the different methods and results will be added. With these modifications, we hope to make the structure clearer, improve the readability, and resolve the reviewers' objections.

Reviewer 1, Jakob Heyman. Reviewer 1's comments focussed primarily on the interpretation of the exposure ages. He wrote "I suggest that you are more open with the fact that the samples may have experienced both prior and incomplete exposure". We thank him for his insight and are enthusiastic to include such considerations in our text. Reviewer 1's main concern is that the exposure age data were treated differently on the

Printer-friendly version

Discussion paper
Interactive comment 
upper lobe and the lower lobe. In a nutshell, if the upper lobe exposure data contain travel time, as shown by the ages becoming older the further away from the headwall scree slope, then consequently the ages on the lower lobe must also contain boulder travel time. This has never been shown before in any other rock glacier cosmogenic nuclide study and we are happy to consider in depth and to express this result. Reviewer 1 suggests that the degree of scatter on the ages on the upper and lower lobes is similar. This has led us to reconsider our interpretation of the exposure ages on the lower lobe and we will change the main interpretation accordingly. We will adopt the same interpretation scheme for all rock-glacier lobes, regardless of their age. Notably, this re-evaluation made us realize that the lower lobe of the rock glacier is not at all relict. The lower lobe is still active today, albeit only marginally. This is remarkable given its early-mid Holocene age and is in itself an important finding. As the actual velocity of the lower lobe can never be known (obtained velocities through aerial image correlation are for today), the amount of travel time contained in the nuclide inventory cannot really be known. We will incorporate such caveats into our revised manuscript. The new approach, that encompasses the fact that the exposure ages for the lower lobe do contain at least some component of travel time, allows the finite-element modelling and velocity determinations to be well merged with the exposure dating results.

Reviewer 1 suggests that we cannot strictly exclude bedrock rockwall pre-exposure. This is true but it is actually true for exposure dating of moraine boulders and landslide boulders. Only years of experience of dating moraines has shown that pre-exposure occurs in less than $2 \%$ of moraine boulders. For rockglaciers, such a body of data does not yet exist. Nevertheless, the fact that rock glaciers, and specifically the talus-derived Bleis Marscha, are fed by active talus production and frequent rock falls provides strong evidence against pre-exposure being acquired in the bedrock wall. Nevertheless, we remain open to this possibility and will cover this point in more detail in our revised version. On the other hand, we relax our previous assumption of 'passive transport' of the boulders on the active rock-glacier surface and readily acknowledge that exposure ages from single boulders may be 'too young' due to shifting or overturning. However,

Printer-friendly version

Discussion paper
Interactive comment 
based on our finding of a - in general - linear relationship between exposure age and down-flow distance allows us to glean information on the timing of development of the rock-glacier lobe from the boulder ages. In a revised manuscript, we will discuss these disturbances and limitations more openly.

Reviewer 2, Leif Anderson. Reviewer 2 brought up several of the same concerns as Reviewer 1 , namely that the age data for the upper lobe cannot be treated differently than the age data for the lower lobe. We also thank him for the additional literature reference on boulder rolling as an important potential process on rock glaciers that we will consult. As described above these points led us to re-assess our interpretation of the ages on the lower lobe.

Reviewer 2 felt that too many details are included in the paper, which hampers its readability and accessibility. To streamline the paper, in the revised version we will delete some of this detail and place related text and figures in the supplemental material. For example, (o) Fourier analysis of surface topography to constrain the viscosity contrast between rock-glacier core and boulder mantle (viscosity ratio estimated with buckle-folding theory), (o) Error assessment of image correlation to estimate the level of detection (correlation of stable reference area in the valley floor), (o) Detailed image correlation results with strain-rate calculations and subdivision into kinematic domains. Similarly, a number of the different lobe classification labels will be deleted, to simplify the paper also in accordance with the re-assessed interpretation of the age of the lower lobe.

Reviewer 2 suspects that the level of detection of the surface velocity we use in the submitted version is too low (currently $5 \mathrm{~cm} / \mathrm{a}$ ) by pointing at the presumably stable offrock glacier slopes that nonetheless show velocities that cluster at $5-10 \mathrm{~cm} / \mathrm{a}$. We partly agree and will discuss possible errors in the pre-processing and orthorectification of the orthophotos. In a revised version, we will adopt a more conservative level of detection of $10 \mathrm{~cm} / \mathrm{a}$. This change does not alter the picture of current Bleis Marscha kinematics. 
Reviewer 2 asked for clarifications on: (i) how the deformation processes are treated in the finite-element model, and (ii) on the assumptions underpinning the construction of the numerical rock-glacier model (3-layer structure). First, all deformation mechanisms, from creep in the ice-rich rock-glacier core to tilting/sliding of the rock fragments in the boulder mantle, are treated as effectively viscous deformation (e.g. the stiff boulder mantle with a much higher viscosity). We will carefully discuss the limitations of this continuum approach. We are confident that this simplified model does capture the processes relevant for the conclusions we draw from it. Second, we agree that we have to separate better the assumptions coming from observations on Bleis Marscha from literature knowledge. More details on the reasoning behind the values chosen for specific parameters will be added either in the main text or in the supplemental section. Reviewer 2 mentions sensitivity tests for the numerical modelling parameters. We will check the relevant rock-glacier modelling literature to get a picture of the range of values chosen in these studies, and do sensitivity tests for poorly constrained, uncertain or disputed values.

We hope that we have provided some constructive comments that address the reviewers' concerns and hope to incorporate these into an eventual future revision. Thank you for your consideration and we look forward to receiving your direction regarding the next steps of review.

Kind regards, Dominik Amschwand, Susan Ivy-Ochs, and Olivia Steinemann on behalf of all co-authors

Interactive comment on The Cryosphere Discuss., https://doi.org/10.5194/tc-2020-209, 2020. 